\title{
Increased Basal REM Sleep But No Difference in Dark Induction or Light Suppression of REM Sleep in Flinders Rats with Cholinergic Supersensitivity
}

\author{
Ruth M. Benca, M.D., Ph.D., David E. Overstreet, Ph.D., Marcia A. Gilliland, Ph.D., \\ Dawn Russell, Bernard M. Bergmann, Ph.D., and William H. Obermeyer, Ph.D.
}

Increased cholinergic sensitivity in the central nervous system has been postulated to account for some of the neuroendocrine abnormalities and sleep disturbances seen in human depressizes. The Flinders Sensitive Line (FSL) rats, which exhibit increased sensitivity to cholinergic agents, have been shown to have REM sleep patterns similar to those seen in depressives, including shorter REM sleep latency and increased daily percentage of REM sleep.

We studied the response of FSL and control rats to brief dark pulses administered during the normal light period (which are known to stimulate REM sleep in albino rats) and to brief light pulses during the normal dark period (which suppress REM sleep in albino rats) to determine whether these responses are affected by central cholinergic hypersensitivity. FSL rats showed REM sleep patterns indistinguishable from controls during light or dark pulse's,

KEY WORDS: REM Sleep; Hypercholinergic Rats; Acetylcholine; Depression; Light

The Flinders Sensitive Line (FSL) and Flinders Resistant Line (FRL) of rats were developed from the Sprague-

From the Department of Psychiatry (RMB; WHO), University of Wisconsin-Madison, Madison, WI; the Department of Psychiatry and Center for Alcohol Studies (DEO), University of North Carolina, Chapel Hill, NC; and the Sleep Research Laboratory (MAG; DR; BMB), The University of Chicago, Chicago, IL.

Address correspondence to Ruth Benca, M.D., Ph.D., Department of Psychiatry, UW Hospitals and Clinics, 600 Highland Avenue, Madison, WI 53792-2475.

Received June 15, 1995; accepted August 1, 1995. which does not support the primary involvement of cholinergic systems in this mechanism of REM sleep regulation.

We also examined REM and non-REM (NREM) sleep patterns in FSL rats and their controls to determine whether they show sleep continuity disturbances or decreased sleep intensity as seen in depression. In agreement with an earlier study, we found that FSL rats had more daily REM sleep and accumulated less NREM sleep between REM bouts than controls. Duration of NREM sleep bouts, total daily NREM sleep time, and EEG amplitude of NREM sleep did not differ between FSL and control rats, suggesting that the cholinergic abnormalities in FSL rats do not produce substantial NREM sleep changes. [Neuropsychopharmacology 15:45-51, 1996]

Dawley line to provide models of differential cholinergic sensitivity based on hypothermic responses to cholinergic agonists (Overstreet 1986). FSL rats show higher cholinergic supersensitivity than FRL rats in two cholinergically mediated behaviors: increased hypothermia in response to oxotremorine sesquifumerate, and increased REM sleep. Cholinergic systems are clearly involved in REM sleep generation: REM sleep is enhanced following the administration of cholinergic agonists in the pons (Hernández-Peón et al. 1963); anticholinergic agents, in contrast, cause significant REM sleep suppression (Domino et al. 1968).

We studied REM sleep regulation by light and dark 
stimulation in FSL rats and control FRL rats to determine whether lights-off triggering of REM sleep is mediated by cholinergic mechanisms. It has been demonstrated previously in albino rats that light inhibits REM sleep (Fishman and Roffwarg 1972) and that transitions from light to dark can stimulate REM sleep episodes (Lisk and Sawyer 1966; Rechtschaffen et al. 1969; Benca et al. 1991; Leung et al. 1992). Since both FSL and FRL rats are albino, exposures to brief dark and light pulses were employed to test susceptibility to REM sleep induction and suppression. If light-dark regulation of REM sleep is mediated by cholinergic mechanisms, FSL rats might be expected to show less suppression of REM sleep during lights-on and/or increased REM sleep triggering following lights-off than FRL rats.

FSL rats have also been proposed as an animal model in which to study the presumed cholinergic abnormalities in depression. A previous study of sleep in FSL rats has demonstrated that, like depressives, FSL rats have a shorter latency from sleep onset to REM sleep and an increased REM percentage of total sleep (Shiromani et al. 1988). It is not known whether the sleep changes in depression result from cholinergic abnormalities alone, although it has been proposed that cholinergic mechanisms may suppress slow-wave sleep (Steriade et al. 1991). We examined uninterrupted sleep in FSL and FRL rats to determine whether non-REM (NREM) sleep abnormalities seen in depressives may also be detected in rats with increased cholinergic sensitivity.

\section{METHODS}

FSL and FRL rats were obtained from the viral-free colony maintained at the University of North Carolina. Both strains were derived from Sprague-Dawley rats based on their responses to the anticholinesterase diisopropylfluorophosphate (DFP) (Overstreet et al. 1979). FSL rats were bred from animals that exhibited a large hypothermic response following DFP challenge, whereas FRL rats were bred from rats with minimal temperature decreases. Rats used in this study were challenged with the cholinergic agonist oxotremorine at the time of weaning and again following physiological recording to confirm their sensitivity or resistance to cholinergic challenge (Overstreet et al. 1992).

Rats aged approximately 100 to 200 days and weighing 400 to $550 \mathrm{~g}$ were anesthetized with ketamine (87 $\mathrm{mg} / \mathrm{kg})$ and xylazine $(13 \mathrm{mg} / \mathrm{kg})$ and implanted with screw EEG electrodes in the skull and silver plate EMG electrodes in the temporalis muscle (Bergmann et al. 1987, 1989).

After at least 1 week of recovery from surgery, rats were placed in individual sound-attenuated recording chambers and connected to recording cables suspended from commutators that allowed free movement. Rats were maintained on a 12-hour light/12-hour dark schedule, with a light intensity between 100 and 150 lux at the cage floor during the light period. Food and water were available ad libitum, and cage temperature was maintained at $28^{\circ} \pm 2^{\circ}$

After 3 to 5 days of adaptation to the recording cages, 4 days of baseline sleep data followed by 4 days of dark pulse stimulation were obtained. Baseline data were obtained for nine animals of each strain. Dark pulse stimulation trials were performed on nine FSL and eight of the 9 FRL animals because the head plug on one FRL animal malfunctioned during the first day of stimulation. Dark pulse stimulation was performed as described previously (Benca et al. 1991). Briefly, cage lights were turned off for 5 minutes of every 30-minute period during the 12-hour light period. Dark pulse trials were begun 3 hours and 25 minutes into the normal light period, except for two rats (one FSL and one FRL) whose triggering trials began 25 minutes into the 12hour light period. The schedules permitted a total of 17 dark pulse trials per day.

An additional four FSL and FRL rats were subjected to light pulse stimulation. Beginning 25 minutes after the start of the 12-hour dark period, cage lights were turned on for 5 of every 30 minutes for a total of 17 trials per day. After four days, the light pulse duration was increased to 10 minutes for 4 days (i.e., rats were exposed to 10 minutes of light beginning every $30 \mathrm{~min}$ utes during the dark period).

Lateral EEG, midline EEG containing hippocampal theta waves, and temporalis EMG were recorded continuously on a Grass polygraph. Signals were also stored in a computerized sleep staging system according to standard techniques for this laboratory (Bergmann et al. 1987). Twenty-four-hour records were scored in 30-s epochs as waking (W), REM sleep, and three substages of NREM sleep distinguished on the basis of EEG amplitude-[low EEG voltage (LS), medium EEG voltage (HS1), or high EEG voltage (HS2)] NREM sleep. All computer scoring was reviewed by visual inspection of polygraph records and overruled on any obvious disagreements (about $0.4 \%$ of epochs). Sleep data were scored by an individual unaware of the strain identities of the rats.

In order to facilitate comparison with an earlier study (Shiromani et al. 1988), we analyzed several parameters (number of REM sleep bouts, REM sleep bout length, REM cycle length, interbout interval, and NREM sleep bout length) by dividing baseline data into 6-hour blocks, beginning with the dark cycle (i.e., quarters 1 and 2 represented the dark period, quarters 3 and 4 represented the light period). The following measures were calculated for each strain by quarter of the day: number of REM sleep bouts (defined as periods of REM sleep interrupted by no more than one 30-s epoch of $W$ or NREM sleep); duration of the REM sleep bouts in 
Table 1. Sleep Stage Percentages for Baseline and Dark Pulse Triggering Days

\begin{tabular}{lcccccc}
\hline & $n$ & Wake & REM & LS & HS1 & HS2 \\
\hline $\begin{array}{l}\text { Baseline } \\
\quad \text { FSL }\end{array}$ & 9 & $42.12 \pm 1.99$ & $7.80 \pm 1.25^{\prime \prime}$ & $2.99 \pm 0.39$ & $26.89 \pm 1.20$ & $20.19 \pm 1.20$ \\
$\quad$ FRL & 9 & $42.28 \pm 3.67$ & $6.47 \pm 0.60$ & $2.58 \pm 0.43$ & $27.78 \pm 2.24$ & $20.88 \pm 2.24$ \\
$\begin{array}{l}\text { Dark pulse } \\
\quad \text { stimulation }\end{array}$ & & & & & & \\
$\quad$ FSL & 9 & $41.60 \pm 2.49$ & $8.06 \pm 1.33^{n}$ & $3.21 \pm 0.29$ & $26.48 \pm 1.49$ & $20.65 \pm 1.91$ \\
$\quad$ FRL & 8 & $42.33 \pm 3.48$ & $6.37 \pm 0.79$ & $2.46 \pm 0.41$ & $27.80 \pm 1.87$ & $21.05 \pm 1.76$ \\
\hline
\end{tabular}

Abbreviations: LS = low-voltage NREM sleep, $\mathrm{HS} 1$ = medium-voltage NREM sleep, HS2 = high-voltage NREM sleep. Wake and sleep stage percentages of total time for 4 days of baseline and 4 days of dark pulse stimulation are expressed as means \pm SEM. Data from only eight FRL rats were available from dark pulse stimulation days because of head-plug failure in one rat.

"FSL rats had significantly more REM sleep than FRL rats at $p<.002$. No other significant stage differences were noted between strains or across conditions.

minutes; REM cycle length (time from the beginning of one REM sleep bout to the beginning of the next REM sleep bout); inter-REM bout interval in minutes (time elapsed from the end of one REM sleep bout to the beginning of the next REM sleep bout); amount of NREM sleep in minutes occurring between REM sleep bouts (measured as interbout interval minus wake time); and duration of NREM sleep bouts in minutes (defined as periods of NREM sleep interrupted by no more than one 30-s epoch of W or REM sleep). EEG amplitudes of REM sleep and NREM sleep in FSL and FRL rats were also compared. Since amplitudes was recorded as the rectified integrated value in arbitrary units for each 30-s epoch, amplitude ratios were calculated. Mean REM or NREM sleep EEG amplitudes were divided by the mean value of wake EEG amplitudes for each rat for each day during baseline. Twenty-four-hour EEG recordings were inspected visually to ensure that no significant artifacts were present in EEG on days used to calculate amplitude ratios. The histograms of EEG amplitude were also examined to verify the absence of significant amounts of outlying data. Sleep parameters were evaluated using analysis of variance (ANOVA), regression analysis, and paired comparison t-tests. REM sleep triggering by dark pulses was considered to be significant if the Wilcoxon signed rank test on REM sleep time for the $5 \mathrm{~min}$ utes of the dark pulse versus the 5 minutes preceding the dark pulse showed a significant increase $(p<.05)$ over all trials for the rat tested. Conversely, REM sleep suppression by light pulses was considered significant if the Wilcoxon signed rank test on REM sleep time for the 5 (or 10) minutes of the light pulse versus the 5 (or 10) minutes preceding the light pulse showed a decrease at $p$ less than .05 over all trials for that rat.

\section{RESULTS}

Twenty-four-hour mean percentages of wake and sleep stages for nine pairs of FSL and FRL rats during base- line and dark pulse stimulation days are shown in Table 1. FSL rats had significantly greater amounts of REM sleep than FRL rats during baseline, a finding consistent with that of a previous report (Shiromani et al. 1988). They also had more REM sleep on dark pulse stimulation days. No significant differences in other stage percentages were observed between FSL and FRL rats, and dark pulse stimulation had no appreciable effects on any total daily sleep stage parameters, including amount of REM sleep.

Sleep architecture during baseline days was examined across the four 6-hour quarters of the day. Both strains had more REM sleep bouts during the two light periods than during the two dark periods $(p<.005)$. Bout lengths in both strains were longer during the light periods $(p<.005)$. FSL rats had significantly more REM sleep bouts across the day than FRL rats (FRL = $50.75 \pm 3.18, \mathrm{FSL}=67.67 \pm 7.99 ; p<.01)$. The mean duration of REM sleep bouts did not differ significantly between the two strains across the day, however (FRL = $1.74 \pm 0.14$ minutes, $\mathrm{FSL}=1.58 \pm 0.21$ minutes).

As expected from their larger number of REM sleep bouts, FSL rats had a shorter REM cycle length $(p<.01$, by repeated-measures ANOVA; data not shown) and showed a trend for shorter time intervals between REM sleep bouts than FRL rats $(p=.061$; Figure 1$)$. Both strains had significantly longer inter-REM intervals in the dark, their usual waking period, than in the light $(p<.02)$. FSL rats showed significantly less NREM sleep between REM sleep bouts across the day than did FRL rats $(F R L=13.20 \pm 2.46$ minutes, $F S L=10.36 \pm$ 2.12 minutes; $p<.02$ ). In both strains, however, the amount of NREM sleep between REM sleep bouts was nearly constant across the day.

Further analysis of NREM sleep was performed by calculating mean duration of NREM episodes across the four 6-hour periods of the day. Although mean lengths of NREM bouts were significantly greater during the light periods $(p<.005)$, no significant differences were noted between the strains across the day. Since EEG am- 

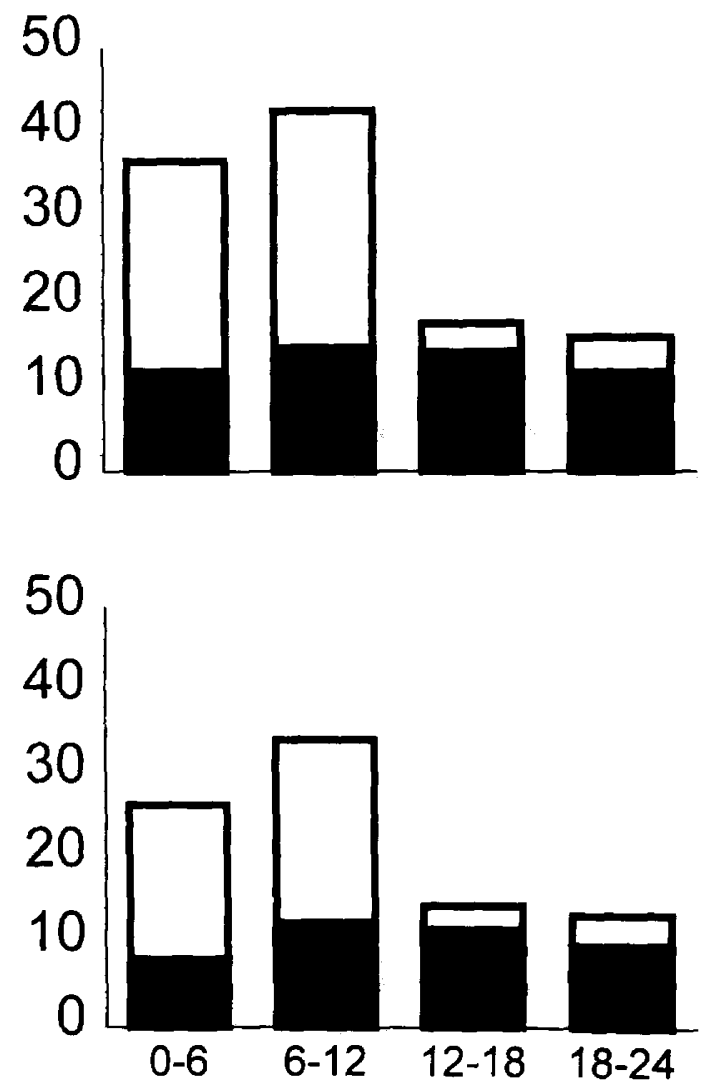

Figure 1. Intervals between REM sleep bouts in minutes expressed as means \pm SEM for FRL (top panel) and FSL (bottom panel) rats for each quarter of the day. Hours 0-12 represent the dark (active) period; hours 12-24 represent the light (rest) period. Inter-REM bout intervals were significantly longer during the dark period for both strains $(p<.001)$. ( $\square$ Wake; NREM) Mean duration of intervals across the day tended to be higher in FRL rats $(p<.061)$. NREM accumulation between REM sleep bouts did not differ across the day within either strain, although FSL rats obtained significantly less NREM sleep between REM sleep bouts than FRL rats across the day $(p<.02)$.

plitude has been described as a measure of sleep intensity, amplitudes of NREM and REM sleep were also compared between strains. There were no differences between strains in the normalized REM or NREM sleep EEG amplitudes; NREM/W amplitude ratios were $1.70 \pm 0.06$ for FSL and $1.70 \pm 0.05$ for FRL rats, and $\mathrm{REM} / \mathrm{W}$ amplitude ratios were $0.99 \pm 0.03$ for FSL and $0.96 \pm 0.02$ for FRL rats.

To determine the effects of increased central cholinergic sensitivity on REM sleep regulation by light-dark stimulation, FSL and FRL rats were subjected to 4 days of dark pulse stimulation. All but one rat of each strain were exposed to dark pulse stimulation during the last 8 hours of the light cycle, since it had been demonstrated that FSL rats obtained proportionately more REM sleep during the latter part of the light cycle as compared to FRL rats (Shiromani et al. 1988). Data from the one FSL and one FRL rat exposed to dark pulses

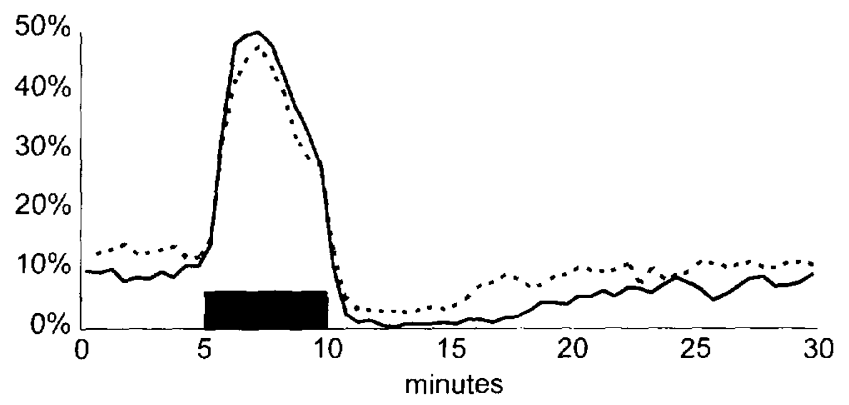

Figure 2. REM sleep percent of time are plotted for 30-s epochs of the 5-minute dark pulses and the light periods immediately preceding and following the dark pulses. Data represent group mean values for the nine FSL (dotted line) and eight FRL (solid line) rats obtained at the standard background light condition (100-150 lux). Shaded bar represents 5-minute lights-off period.

during the first 8 hours of the light cycle were not significantly different from their respective groups, so data from all animals within each strain were combined. All FRL rats and all but one FSL rat showed significant REM sleep induction by dark pulses when background light intensity was 100 to 150 lux; the single nontriggering FSL rat showed significant REM sleep induction by dark pulses at a higher background light intensity $(2,500$ lux; results not shown). For the purposes of comparing the two strains under similar conditions, only data obtained under standard illumination (100-150 lux) are reported. As shown in Figure 2 and Table 2, FSL rats showed a trend toward greater REM sleep amounts in the 5-minute periods immediately preceding and following the dark pulses than did FRL rats, but these differences did not reach statistical significance. Repeatedmeasures ANOVA of REM sleep amounts in the 5-minute dark pulses and the 5-minute light periods immediately preceding and following the dark pulses revealed no significant difference between strains or any significant interaction of strain with period. Both strains showed significant increases in REM sleep during the dark pulses and significant suppression of REM sleep in the

Table 2. Dark Pulse Triggering of REM Sleep

\begin{tabular}{lrrrr}
\hline \multicolumn{1}{c}{ Strain } & $\begin{array}{r}\text { Preceding } \\
\text { 5 Minutes } \\
\text { Lights-On }\end{array}$ & $\begin{array}{c}\text { 5-Minute } \\
\text { Dark Pulse }\end{array}$ & $\begin{array}{c}\text { Following } \\
\text { 5-Minutes } \\
\text { Lights-On }\end{array}$ \\
\hline REM \% of Total Time & & & & \\
$\quad$ FSL & 9 & $13.7 \pm 6.0$ & $35.2 \pm 7.6$ & $4.7 \pm 3.1$ \\
$\quad$ FRL & 8 & $8.9 \pm 3.3$ & $38.0 \pm 6.6$ & $1.9 \pm 1.2$ \\
REM \% of Total Sleep & & & & \\
$\quad$ FSL & 9 & $16.1 \pm 6.6$ & $45.1 \pm 10.0$ & $6.9 \pm 3.9$ \\
FRL & 8 & $11.4 \pm 4.2$ & $48.9 \pm 7.3$ & $3.2 \pm 2.1$ \\
\hline
\end{tabular}

Values expressed as mean $\pm S D$. Within each strain, REM sleep percentages (of both total time and total sleep) differed significantly $(p<.01$ after Bonferroni correction) from REM sleep percentages in the other 5-minute periods. 
lights-on periods immediately following the dark pulses in comparison to the preceding 5-minute lightson periods (Table 2; Figure 2).

To assess further whether REM sleep inhibition by light differs in FSL versus FRL rats, four additional pairs of animals were subjected to 5- and 10-minute light pulses during the 12-hour dark period. As seen in Figure 3, REM sleep amounts before, during, and after light pulses did not differ significantly between strains, as determined by repeated-measures ANOVA of REM sleep amounts during the 5- or 10-minute light pulses compared to the corresponding equal-length dark periods immediately preceding and following the light pulses. REM sleep increases in the lights-off periods immediately following light pulses were smaller after 5 minutes than after 10 minutes of light pulses in both FSL and FRL rats.

\section{DISCUSSION}

A previous study of sleep patterns in FSL rats has shown that they obtain more REM sleep as a percentage of total sleep time, enter REM sleep more rapidly from NREM sleep, and have a shorter REM sleep cycle length (time between the beginnings of two successive REM sleep bouts) in comparison with control FRL rats (Shiromani et al. 1988). It has been suggested that increased
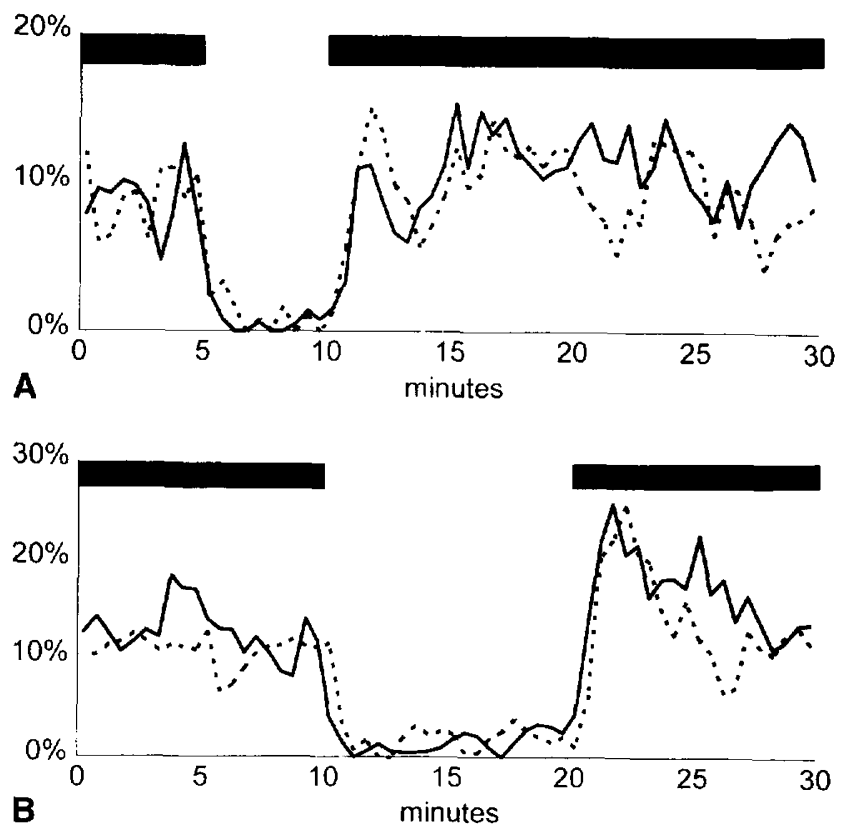

Figure 3. REM sleep percent of time are plotted for 30-second epochs of the 5-minute (A) or 10-minute (B) light pulses and the dark periods immediately preceding and following the light pulses. Data represent group mean values for four FSL (dotted line) and four FRL (solid line) rats. Light intensity was 100-150 lux during lights-on. Shaded bar indicates lightsoff periods. numbers of central muscarinic receptors may lead to acceleration of the REM sleep cycle, and thus to increases in overall REM sleep amounts.

We investigated normal sleep patterns in FSL and FRL rats in a 12-hour light/12-hour dark cycle and assessed their responses to REM sleep stimulation by dark pulses and suppression by light pulses. In agreement with Shiromani et al. (1988), we found that during baseline conditions FSL rats exhibited significantly greater REM sleep percent of total time as well as of total sleep; no strain differences were observed in wake or NREM sleep parameters. Further analysis of sleep cycles across the 24-hour period in the two strains confirmed that FSL rats had significantly more bouts of REM sleep across the day, whereas REM sleep bout lengths were comparable between the two strains.

To investigate the nature of the reduced REM sleep cycle length, we analyzed the time periods between REM sleep bouts across the day. Although FSL rats showed a tendency for shorter inter-REM bout intervals, the difference between strains did not reach significance. However, the accumulation of NREM sleep between REM sleep bouts was significantly lower in FSL than in FRL rats. Furthermore, the amounts of NREM sleep obtained between REM sleep bouts remained constant across the day within each strain, although interREM bout intervals lengthened dramatically during the 12-hour active period. These findings are consistent with the reduced REM sleep cycle length and shorter latency to REM sleep in FSL rats reported earlier (Shiromani et al. 1988).

It does not appear that the differences in REM sleep cycle length can be accounted for by differences in pressure for NREM sleep per se, since daily NREM times were similar in FSL and FRL rats. However, there could be differences between FSL and FRL rats in the quality of NREM sleep, which might account for the differences in REM-NREM sleep relationships. Quality of NREM sleep is often defined as some function of EEG amplitude and time (Borbély 1987), and previous studies have used EEG amplitude as a measure of sleep intensity in rats (Mistlberger et al. 1987a, and 1987b). To compare NREM sleep intensity in FSL versus FRL rats, EEG amplitude of NREM sleep was calculated in relationship to waking amplitude. No differences were observed between strains. NREM sleep was not more fragmented in FSL rats, as indicated by the similar mean durations in NREM sleep bouts in FSL and FRL rats. Thus, the data presented here do not suggest that primary deficits in NREM sleep in FSL rats accounts for the observed changes in REM sleep behavior. Rather, they indicate a positive, albeit not identical, relationship between NREM and REM sleep expression in the two strains.

We studied the effects of dark pulse stimulation on REM sleep in FSL and FRL rats, as the transition from 
light to dark has been shown to cause a rapid induction of REM in albino rats (Benca et al. 1991; Leung et al. 1992). It was of interest to determine whether FSL rats would show increased triggering of REM sleep compared to FRL rats in response to dark pulses, which would both support the hypothesis that REM sleep is more easily induced in FSL rats and suggest the involvement of a cholinergic mechanism in this behavior. Surprisingly, during the 5-minute dark pulses the rates of REM sleep induction and the overall amounts of REM sleep obtained were similar in FSL and FRL rats. FSL rats, however, showed a trend toward higher mean percentages of REM sleep in the 5-minute periods before and after dark pulses, in keeping with their higher overall REM sleep percentages. That these differences did not reach significance may be due to the inherent conservatism of the Bonferroni correction, as well as perturbations of the system caused by massive REM sleep increases during dark pulse triggering. There were no significant strain differences in the magnitudes of the REM sleep triggering responses. One possible explanation for this phenomenon is that REM sleep induction following lights-off may in part represent release from REM sleep suppression by light. The relatively long periods of light exposure in the dark pulse paradigm could have caused maximum induction of REM sleep "need" in both strains, resulting in a ceiling effect during the triggering period, thus making it impossible to detect strain differences in REM sleep responses to dark pulses. If REM sleep "need" builds up at a faster rate in FSL rats, greater REM sleep triggering in FSL vs. FRL rats might be expected following shorter light pulses. Therefore, FSL and FRL rats were exposed to 5-minute and 10-minute light pulses to assess the REM sleep-suppressive effects of light as well as REM sleep rebound following lights-off stimulation. REM sleep was equivalently suppressed during light pulses in both strains, and no differences in REM sleep triggering by lights-off were observed. REM sleep immediately following 5-minute light pulses was not significantly higher than in the dark periods immediately preceding the light pulses in either strain. Significant increases in REM sleep above levels seen during the dark periods preceding light pulses were observed in both strains after 10-minute light pulses $(p<.005)$, a finding consistent with the hypothesis that lights-off REM sleep triggering above baseline levels normally seen during light periods may depend at least in part on prior REM sleep suppression by light.

FSL rats were originally developed to model the cholinergic abnormalities in human depressives. As such, they provide a paradigm in which to study the role of the cholinergic system in a variety of behavioral changes associated with depression, including sleep abnormalities. However, they do not appear to show the deficits in slow-wave sleep (as would be indicated by decreased EEG amplitude) or sleep continuity disruptions (such as decreased total sleep time, prolonged sleep latency, or reduced sleep efficiency) usually seen in depression (reviewed in Benca et al. 1992). It is possible that these latter abnormalities are not related to cholinergic hypersensitivity, although recent studies have suggested that cholinergic activity may suppress delta activity in NREM sleep (Steriade et al. 1991). There is no evidence to date that the FSL rats exhibit abnormalities in the noradrenergic system, which could be relevant to the lack of NREM sleep abnormalities (Overstreet 1993; Overstreet and Janowksy 1991).

Alternatively, rats may not express the full range of sleep abnormalities seen in humans. Longitudinal studies of sleep in patients during depression and remission have suggested that sleep abnormalities may relate to both the acute state of depression and trait like features that persist during clinical remission (Hauri et al. 1974; Coble et al. 1979; Rush et al. 1986). It is not known whether FSL rats model acute depression or the more trait like components of depression, and which of the two relates to increased cholinergic sensitivity.

The physiological mechanisms by which increased muscarinic receptor density in FSL rats leads to increases in REM sleep are not known. REM sleep triggering by dark pulses and REM sleep suppression by light, however, were not different in FSL and FRL rats. The results suggest that the mechanism of REM sleep regulation by light-dark stimulation may not be identical to normal REM sleep induction. Furthermore, light-dark regulation of REM sleep in albino rats may not be mediated primarily by cholinergic systems and/or may be unaffected by cholinergic hypersensitivity.

\section{ACKNOWLEDGMENTS}

The authors gratefully acknowledge the contributions of Jean Shin. This work was supported by NIH grant NS27730 and NIMH grant MH4151.

\section{REFERENCES}

Benca RM, Bergmann BM, Leung C, Nummy D, Rechtschaffen A (1991): Rat strain differences in response to dark pulse triggering of paradoxical sleep. Physiol Behav 49:83-87

Benca RM, Obermeyer WH, Thisted RA, Gillin JC (1992): Sleep and psychiatric disorders: A meta-analysis. Arch Gen Psychiatr 29:651-668

Bergmann BM, Winter JB, Rosenberg RS, Rechtschaffen A (1987): NREM sleep with low-voltage EEG in the rat. Sleep 10:1-11

Bergmann BM, Kushida CA, Everson CA, Gilliland MA, Obermeyer WH, Rechtschaffen A (1989): Sleep deprivation in the rat: II. Methodology. Sleep 12:5-12 
Borbély AA (1987): The S-deficiency hypothesis of depression and the two-process model of sleep regulation. Pharmacopsychiatry 20:23-29

Coble PA, Kupfer DJ, Spiker DG, Neil JF, McPartland RJ (1979): EEG sleep in primary depression: A longitudinal placebo study. J Affective Disorders 1:131-138

Domino EF, Yamamoto K, Dren AT (1968): Role of cholinergic mechanisms in states of wakefulness and sleep. Prog Brain Res 28:113-133

Fishman R, Roffwarg HP (1972): REM sleep inhibition by light in the albino rat. Exp Neurol 36:166-178

Hauri PJ, Chernik D, Hawkins DR, Mendels J (1974): Sleep of depressed patients in remission. Arch Gen Psychiatr 31:386-391

Hernández-Peón R, Chávez-lbarra G, Morgane PJ, TimoIaria C (1963): Limbic cholinergic pathways involved in sleep and emotional behavior. Exp Neurology 8:93-111

Kupfer DJ, Gillin IC, Coble PA, Spiker DG, Shaw DH, Holzer B (1981): REM sleep, naps, and depression. Psychiatr Res 5:195-203

Leung C, Bergmann BM, Rechtschaffen A, Benca RM (1992): Heritability of dark pulse triggering in paradoxical sleep in rats. Physiol Behav 52:127-131

Lisk RD, Sawyer CH (1966): Induction of paradoxical sleep by lights-off stimulation. Proc Soc Exp Biol Med 123: 664-667

Mistlberger RE, Bergmann BM, Rechtschaffen A (1987a): Relationships among wake episode lengths, contiguous sleep episode lengths, and electroencephalographic delta waves in rats with suprachiasmatic nuclei lesions. Sleep $10: 12-24$

Mistelberger R, Bergmann B, Rechtschaffen A (1987b): Period-amplitude analysis of rat electroencephalogram: Effects of sleep deprivation and exercise. Sleep 10:508-522
Overstreet DH (1986): Selective breeding for increased cholinergic function: Development of a new animal model of depression. Biol Psychiatr 21:49-58

Overstreet DH (1993): The Flinders Sensitive Line rat: A genetic model of depression. Neurosci Biobehav Rev $17: 51-68$

Overstreet DH, Janowsky DS (1991): A cholinergic supersensitive model of depression. In Boulton A, Baker G, Martin Iverson $M$ (eds), Neuromethods, vol 19: Animal Models in Psychiatry II. Clifton NJ, Human Press, 81-114

Overstreet DH, Russell RW, Helps SC, Messenger M (1979): Selective breeding for differences in sensitivity to the anticholinesterase, DFP. Psychopharmacology 65:15-20

Overstreet DH, Rezvani AH, Janowsky DS (1992): Genetic animal models of depression and ethanol preference provide support for cholinergic and serotonergic involvement in depression and alcoholism. Biol Psychiatr 31: 919-936

Rechtschaffen A, Dates R, Tobias M, Whitehead WE (1969): The effect of lights-off stimulation on the distribution of paradoxical sleep in the rat. Comm Behav Biol 3:93-99

Rush AJ, Erman MK, Giles DE, Schlesser MA, Carpenter G, Vasavada N, Roffwarg HP (1986): Polysomnographic findings in recently drug-free and clinically remitted depressed patients. Arch Gen Psychiatr 43:878-884

Shiromani PJ, Overstreet DH, Levy D, Goodrich CA, Campbell SS, Gillin JC (1988): Increased REM sleep in rats selectively bred for cholinergic hyperactivity. Neuropsychopharmacology 1(2):127-133

Steriade M, Curro Dossi RC, Nunez A (1991): Network modulation of a slow intrinsic oscillation of cat thalamocortical neurons implicated in sleep delta waves: Cortically induced synchronization and brainstem cholinergic suppression. J Neurosci 11:3200-3217 